\title{
COMPUTATIONAL AND EXPERIMENTAL DEVELOPMENT OF NOVEL HIGH TEMPERATURE ALLOYS
}

\author{
M.J. Kramer, P.K. Ray, T. Brammer and M. Akinc \\ Ames Laboratory and Department of Materials Science and Engineering, \\ Iowa State University, Ames, Iowa 50011
}

\begin{abstract}
The work done in this paper is based on our earlier work on developing an extended Miedema model and then using it to downselect potential alloy systems. Our approach is to closely couple the semi-empirical methodologies to more accurate ab initio methods to identify the best candidates for ternary alloying additions. The architectural framework for our material's design is a refractory base metal with a high temperature intermetallic which provides both high temperature creep strength and a source of oxidatively stable elements. Potential refractory base metals are groups IIIA, IVA and VA. For Fossil applications, Ni-Al appears to be the best choice to provide the source of oxidatively stable elements but this system requires a 'boost' in melting temperatures to be a viable candidate in the ultra-high temperature regime $\left(>1200^{\circ} \mathrm{C}\right)$. Some late transition metals and noble elements are known to increase the melting temperature of $\mathrm{Ni}-\mathrm{Al}$ phases. Such an approach suggested that a Mo-Ni-Al system would be a good base alloy system that could be further improved upon by adding Platinum group metals (PGMs). In this paper, we demonstrate the variety of microstructures that can be synthesized for the base alloy system, its oxidation behavior as well as the oxidation behavior of the PGM substituted oxidation resistant $B 2 \mathrm{NiAl}$ phase.
\end{abstract}

\section{INTRODUCTION}

Increases in operating temperatures of gas turbines are expected to result in increased Carnot efficiencies. An increase in operating temperatures entails with it an inherent loss in creep strength and significantly elevated oxidation rates. Additionally, the alloys in the gas turbines in power generation industries are exposed to coal combustion environment, resulting in exposure to steam, $\mathrm{CO}, \mathrm{SO}_{\mathrm{x}}, \mathrm{NO}_{\mathrm{x}}$ etc. Hence, the key challenge in high temperature alloy industry is the development of alloys that can function at higher and higher temperatures so that the balance between life-time and down-time stays positive [1].

Ni-based superalloys with a corresponding $\mathrm{Ni}$-Al based coating is used for service temperatures in excess of $1000^{\circ} \mathrm{C}$. Commercial overlay coatings are typically MCrAlY alloys, whereas diffusion coatings are based on $\beta$-NiAl. The choice of coating materials is dictated by their ability to form strong bonding with the substrate, formation of an adherent slow-growing 
thermally grown oxide (TGO), and high temperature thermodynamic and microstructural stability [2]. Maximum temperature capability of current commercial coatings is limited to $1150^{\circ} \mathrm{C}$. However, the objectives of FutureGen project require alloys that can function at about $1350^{\circ} \mathrm{C}$. Hence, design of ultra high temperature alloys is a major task in DOE NETL's FutureGen gas turbine program. Such large increases in operating temperatures will require new materials and new engineering. An Edisonian approach to the discovery of new materials is tedious and we lack the numerical tools to efficiently predict new phases. A rapid means of 'sieving' through this phase space to identify the most promising combinations is necessary.

We attempt to tackle this problem by taking an integrated approach by combining the speed of semi-empirical calculations, with the accuracy of ab initio studies and validate the computational approach with targeted experimental studies. In this paper, we use this integrated approach to study the role of transition metal additions to the $B 2 \mathrm{NiAl}$ alloy and study the oxidation behavior of the downselected base alloy.

\section{THEORY}

The semi-empirical calculations were performed using an extended Miedema approach. This approach is based on estimation of formation enthalpies of constituent binary systems and then assessing the ternary formation enthalpies as a weighted sum of binaries, with the weights being decided on the basis of energy minimization under conditions of mass balance. Details of this approach can be found in Ray et al. [3].

The base alloy system was chosen based on the difference in formation enthalpy between the $B 2$ $\mathrm{NiAl}$ and the corresponding transition metal aluminide. A larger difference in formation enthalpies, with the $B 2 \mathrm{NiAl}$ having a lower formation enthalpy, indicates a possibility of a metal rich solid solution $+B 2$ phase field. This would conform to our target architectural framework with the solid solution being responsible for ductility and the $B 2 \mathrm{NiAl}$ being responsible for oxidation resistance.

Semi-empirical calculations on the changes in formation enthalpies due to alloying additions to the $B 2 \mathrm{NiAl}$ phase was studied using Miedema's model. Figure 1 shows the result of these calculations for a 5 atom $\%$ addition of the transition metal for Ni site. Similar calculations were carried out over 0-15 atom\% range at 1 atom\% intervals for both $\mathrm{Ni}$ and $\mathrm{Al}$. Based on the results from these calculations, additions were chosen such that they would readily alloy with the intermetallic while not reacting with the transition metal framework.

The ab initio calculations were carried out using the Vienna ab initio Simulation Package (VASP) [4-6] to get the thermodynamically stable structure of $B 2$ phase in the $\mathrm{Ni}_{1-\mathrm{x}} \mathrm{AlTM}_{\mathrm{x}}$ and $\mathrm{NiAl}_{1-\mathrm{x}} \mathrm{TM}_{\mathrm{x}}$ systems using a 54-atom supercell, where TM represents the transition metal element selected by the Miedema's model. The density functional calculations are performed using a plane-wave pseudo-potential representation, with ultra-soft pseudo-potentials [5] for all species and with a plane-wave energy cutoff of $300 \mathrm{eV}$. The $k$-point sampling was chosen to 
converge all of the total energies to an accuracy of $2 \mathrm{meV} /$ atom. The $k$-point grid used for structural relaxation was $6 \times 6 \times 6$, chosen according to the Monkhorst-Pack scheme [7], and symmetry reduced to the irreducible Brillouin zone.

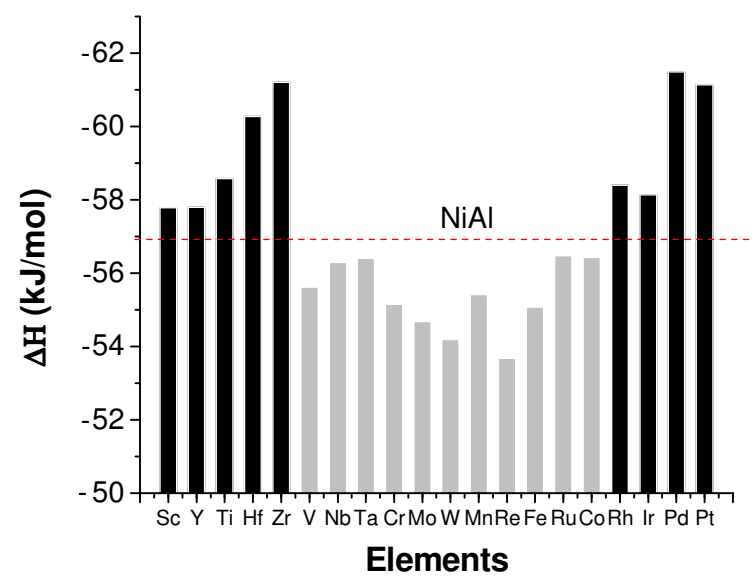

Figure 1. Formation enthalpies calculated using the extended Miedema model for different alloying additions with a nominal composition $\mathrm{Ni}_{45} \mathrm{Al}_{50} \mathrm{TM}_{5}$, where $\mathrm{TM}$ is the transition metal addition. The dashed line indicates the formation enthalpy of $\mathrm{NiAl}$ using Miedema's model without any alloying additions.

\section{EXPERIMENTAL DETAILS}

Semi-empirical Miedema calculations indicated Mo-Ni-Al as a good candidate for high temperature applications. A number of compositions were then studied within the Mo-Ni-Al system to assess the system experimentally. Mo-Ni-Al alloys are known to exhibit a two phase (bcc $\mathrm{Mo}+\beta-\mathrm{NiAl})$ microstructure over a limited range of compositions [8]. All the experiments in the present study were carried out in this phase field. Higher phase fraction of Molybdenum would be detrimental to the oxidation resistance; hence the phase fraction of Mo was kept below 35 at\% in all cases. The alloys were synthesized using powder metallurgical techniques as well as casting.

The powder metallurgy involved mixing the pure metal powders $(\sim 10 \mu \mathrm{m})$ in a mixer/shaker (SPEX 8000, SPEX CertiPrep Inc, Metuchen, NJ) for 10 minutes followed by compaction of the powders by dry pressing at a pressure of $3500 \mathrm{psi}(24 \mathrm{MPa})$. The cylindrical pellets thus produced had a diameter of $10 \mathrm{~mm}$ and a height of $20 \mathrm{~mm}$. The pellets were then sintered at $1750^{\circ} \mathrm{C}$ for 45 minutes. The pellets synthesized by this route had nominal compositions of $\mathrm{Mo}=$ $15,20,25$ and 35 at\%, with the balance in each case being equiatomic NiAl. Oxidation coupons having a diameter of $10 \mathrm{~mm}$ and a thickness of $1 \mathrm{~mm}$ were cut from the cylindrical samples and subjected to interrupted oxidation tests at $1100^{\circ} \mathrm{C}$. Oxidation tests with multiple compositions allowed us to study the effect of Mo phase fraction and determine an appropriate composition range where the oxidation resistance was reasonably optimized.

The composition of the drop cast alloy $\left(\mathrm{Mo}_{20} \mathrm{Ni}_{40} \mathrm{Al}_{40}\right)$ was decided based on the oxidation behavior of the sintered alloys. Drop cast samples were prepared from pelletized elemental 
powder mixture of Mo (Alfa Aesar, 99.5\% purity), Ni (MPC, 99.6\% purity) and Al (Alfa Aesar, $99.8 \%$ purity) which were arc-melted. The alloys were re-melted thrice to achieve a greater degree of homogenization followed by drop-casting in order to obtain a cylindrical rod. The drop-cast alloy was subject to interrupted oxidation tests over a range of temperatures from $1000^{\circ} \mathrm{C}$ to $1200^{\circ} \mathrm{C}$. In this test, the samples were exposed to the target temperatures for a total of 20 hours with occasional interruptions for mass measurements at ambient temperature. The testing temperatures were deliberately kept at $1200^{\circ} \mathrm{C}$ or lower since our work on oxidation of pure nickel aluminides at temperatures above $1200^{\circ} \mathrm{C}$ (discussed below) has shown massive scale spallation. Microstructures of the as-prepared alloys as well as the oxidized coupons were studied using a JEOL 5910LV scanning electron microscope (JEOL, Tokyo, Japan) at an accelerating voltage of $20 \mathrm{kV}$.

The relatively poor oxidation behavior of the nickel aluminide beyond $1200^{\circ} \mathrm{C}$ necessitated careful alloying additions to the $\mathrm{NiAl}$ phase in order to increase its operating temperature. The $\mathrm{Ni}$-Al-TM alloys were produced from pieces cut from pure bulk metal sheets obtained from the Materials Preparation Center at Ames Laboratory, having a purity of $99.6 \%$ or more. Pure Ni and Al were first arc-melted together in an argon atmosphere to form $\beta$-NiAl. The ab initio calculations suggested that the transition metals have a preference for the Ni site in the $B 2$ structure. Alloys with compositions $\mathrm{Ni}_{50-\mathrm{x}} \mathrm{Al}_{50} \mathrm{TM}_{\mathrm{x}},(\mathrm{x}=3,6$ and 9) were then arc-melted in an argon atmosphere. The samples were re-melted three times to achieve better homogenization before drop-casting. The drop-cast rods were annealed at $1300^{\circ} \mathrm{C}$ in an argon atmosphere for 6 hours to ensure homogeneity. SEM was used to characterize the microstructure while phase analysis was done using x-ray diffraction (XRD) on a Philips PANalytical x-ray diffractometer (Panalytical, Almelo, Netherlands) with a Bragg-Brentano geometry and $\mathrm{Cu} \mathrm{K} \alpha_{1}$ radiation $(\lambda=$ $1.54056 \AA$ ). The $\mathrm{x}$-ray data was Rietveld refined using the GSAS software in order to estimate the lattice parameters of the phases as well as determine site preference for different elements [9]. Of the transition metals studied, it was observed that alloys with platinum group metal (PGM) modifications formed single phase alloys while the other alloys showed a eutectic microstructure. Hence, further studies were carried out only on the PGM modified alloys. Oxidation coupons were prepared as described above, but more aggressive testing conditions, $1300^{\circ} \mathrm{C}$ for two hours and ambient for $30 \mathrm{~min}$, were employed to determine if the PGM improved the oxidation stability of $\mathrm{NiAl}$ reservoir phase.

\section{RESULTS AND DISCUSSION}

Liquid phase sintered samples showed a strong composition dependence of oxidation. Alloys under 20 at\% Mo, with equiatomic NiAl showed fairly good oxidation resistance, while the alloys with a higher volume fraction of $\mathrm{Mo}$ (e.g. $\mathrm{Mo}_{35} \mathrm{Ni}_{32.5} \mathrm{Al}_{32.5}$ ) failed to form a passivating oxide layer (Fig. 2). The strong composition dependence of oxidation is due to Mo's deleterious effect on the oxidation resistance. Minimizing the inter-connected Mo network is required. 


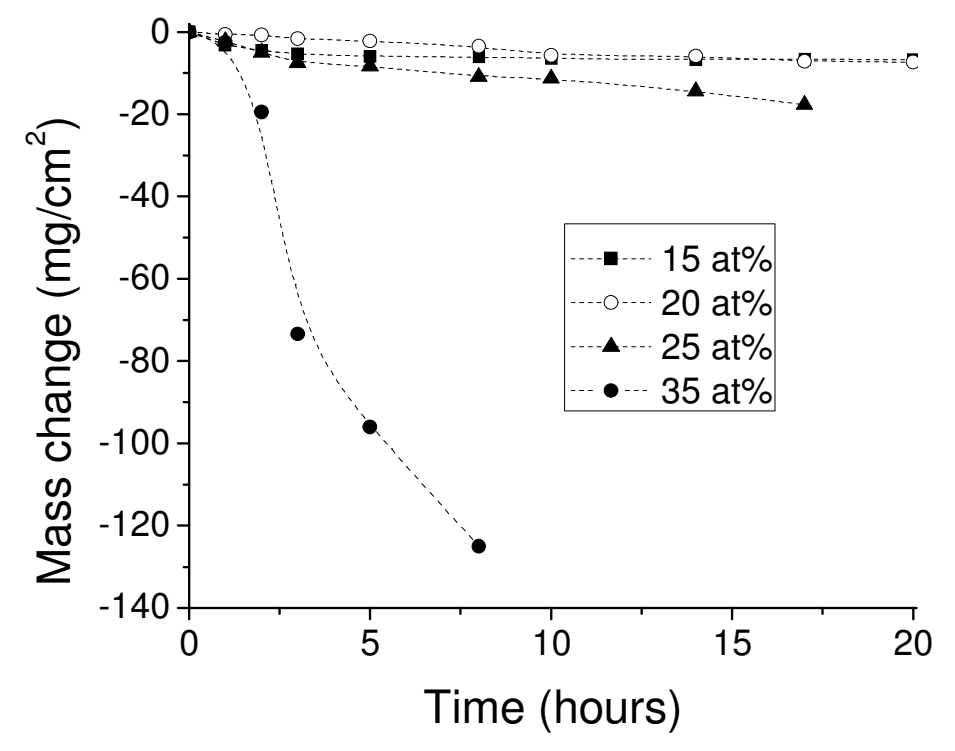

Figure 2: Interrupted flowing air oxidation of liquid phase sintered alloys of different Mo content at $1100^{\circ} \mathrm{C}$.

Figure $3 \mathrm{a}$ shows a low magnification microstructure of the oxidized $\mathrm{Mo}_{20} \mathrm{Ni}_{40} \mathrm{Al}_{40}$ cast alloy at $1200^{\circ} \mathrm{C}$. It can be seen that the alloy has developed a continuous alumina scale of approximately $5 \mu \mathrm{m}$ thickness, across the length of the sample. A closer look at the oxide scale at higher magnification (Figure 3b) shows that the scale thickness fluctuations is due to presence of long Mo dendrites growing into the alloy.
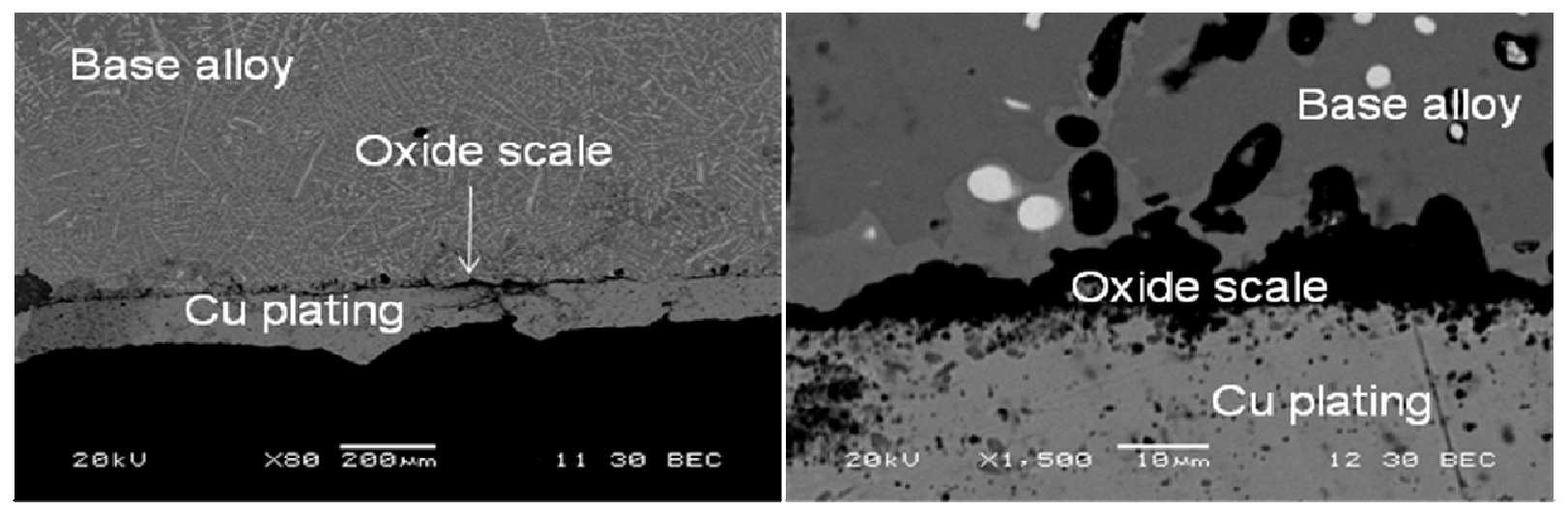

Figure 3: (a) Low magnification image of the oxidized alloy. The alumina scale appears to have formed continuously on the surface. (b) Higher magnification image of the oxidized alloy showing the non-uniformity of scale thickness primarily as a result of presence of Mo phase at the surface. Mo gets oxidized and the resultant $\mathrm{MoO}_{3}$ volatalizes allowing oxygen to penetrate relatively larger distances into the alloy.

It has been widely observed in case of nickel based alloys that the initial oxidation product of $\mathrm{NiAl}$ is the formation of $\mathrm{NiO}$ [10]. Subsequently, $\mathrm{NiO}$ is reduced by $\mathrm{Al}$ resulting in the formation of $\mathrm{Ni}$ and $\mathrm{Al}_{2} \mathrm{O}_{3}$. Typically such a reduction results in linear rather than parabolic kinetics. Aluminum can also react with atmospheric oxygen to form $\mathrm{Al}_{2} \mathrm{O}_{3}$ which should exhibit parabolic oxidation kinetics. Additionally, $\mathrm{NiO}$ and $\mathrm{Al}_{2} \mathrm{O}_{3}$ can react to form a $\mathrm{NiAl}_{2} \mathrm{O}_{4}$ spinel [11]. All the 
reactions involving $\mathrm{Ni}$ and $\mathrm{Al}$ result in a slow mass gain, whereas the oxidation of Mo and the subsequent volatilization of $\mathrm{MoO}_{3}$ results in a rapid mass loss [12]. Consequently, the overall oxidation rate is a sum of mass gain due to oxidation of $\mathrm{Ni}$ and $\mathrm{Al}$ and loss due to volatilization of $\mathrm{MoO}_{3}$. Figure 4 shows the oxidation kinetics during the interrupted oxidation process for the $\mathrm{Mo}_{20} \mathrm{Ni}_{40} \mathrm{Al}_{40}$ alloy at $1200^{\circ} \mathrm{C}$. As a baseline comparison the steady state mass loss value for the $T 2-\mathrm{Mo}_{5} \mathrm{SiB}_{2}$ compound is shown on the same figure for comparison. The preliminary data indicates that the oxidation behavior of Mo-Ni-Al alloy is comparable to the refractory metal silicides at fairly elevated temperatures.

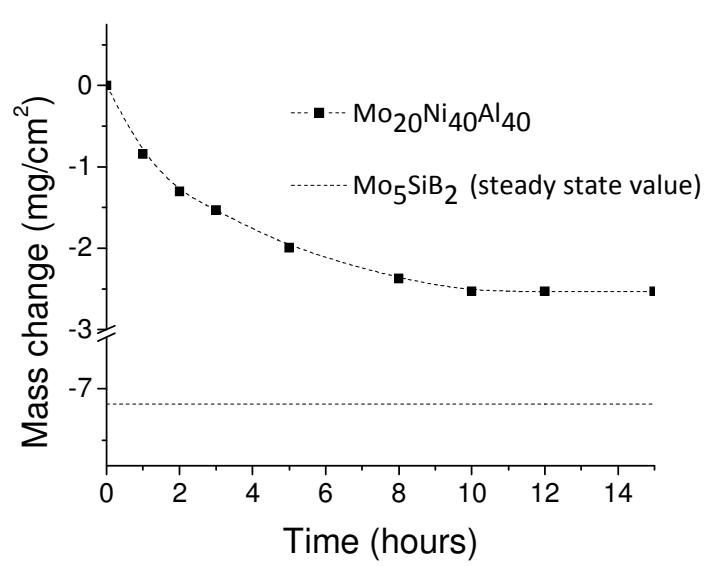

Figure 4: Interrupted flowing air oxidation of an arcmelted and drop cast alloy $\left(\mathrm{Mo}_{20} \mathrm{Ni}_{40} \mathrm{Al}_{40}\right)$ at $1200^{\circ} \mathrm{C}$

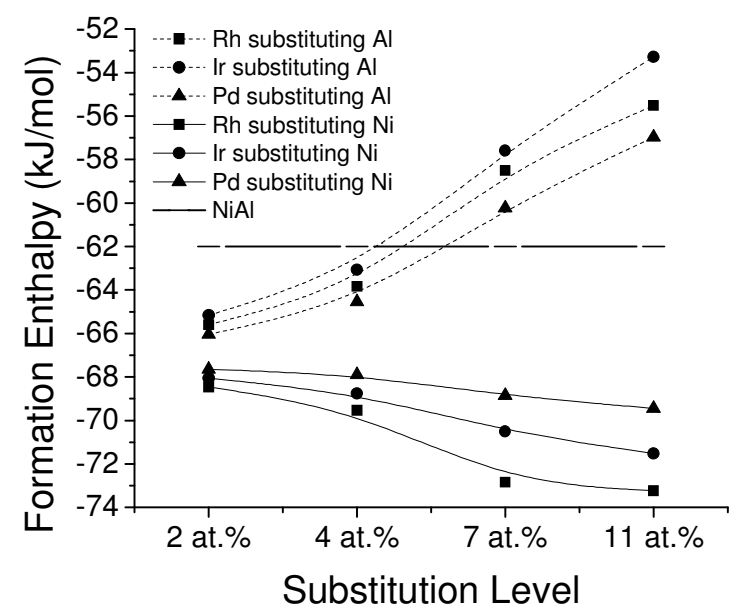

Figure 5: Variation of formation enthalpy of $\beta-\mathrm{NiAl}$ with PGM additions.

Figure 5 shows the variation of formation enthalpy of PGM substituted $\beta$-NiAl. It can be seen that substitution of PGM elements for Ni is energetically favorable. Also, controlled additions result in a monotonic change in formation enthalpy. This suggests that these PGM additions might be beneficial for obtaining an increase in the melting temperature of the nickel aluminide. The other transition metals that showed large negative formation enthalpies with the nickelaluminide included $\mathrm{Hf}, \mathrm{Y}$, and Zr. All of these additions were experimentally found to produce deep eutectics (see Figure 6). The formation enthalpy of these eutectics showed them to be quite stable, but the two phase eutectic is not desired for high temperature applications. All of the PGM additions formed a single phase solid solution with the $\beta$-NiAl.

Figure 7 shows the oxidation kinetics for the three PGM substitutions (all 6 at $\%$ ) to the $\beta$-NiAl compared with the $\beta$-NiAl as a baseline. It can clearly be seen that the Pd substitution performed worse than the $\beta$-NiAl during oxidation testing at $1300^{\circ} \mathrm{C}$. The oxide spallation of the $\mathrm{Pd}$ sample was noticeable in the early stages of the test. The spallation flakes were large and discernable with the naked eye, just like the $\beta$-NiAl spallation. The $\mathrm{Rh}$ sample performed better than the base alloy $\mathrm{NiAl}$, but worse than the alloy doped with Ir. The sample with Rh showed an adherent oxide scale initially, but after five hours oxide spallation was observed. From the oxidation resistance point of view Ir substitution to $\beta$-NiAl appears to be superior. No sign of spallation was evident at times shorter than 20 hours for alloys with 9 at\% dopant. In all cases it should be noted that the highest performing alloy at the end of 20 hours was always the alloy with highest ternary substitution. 


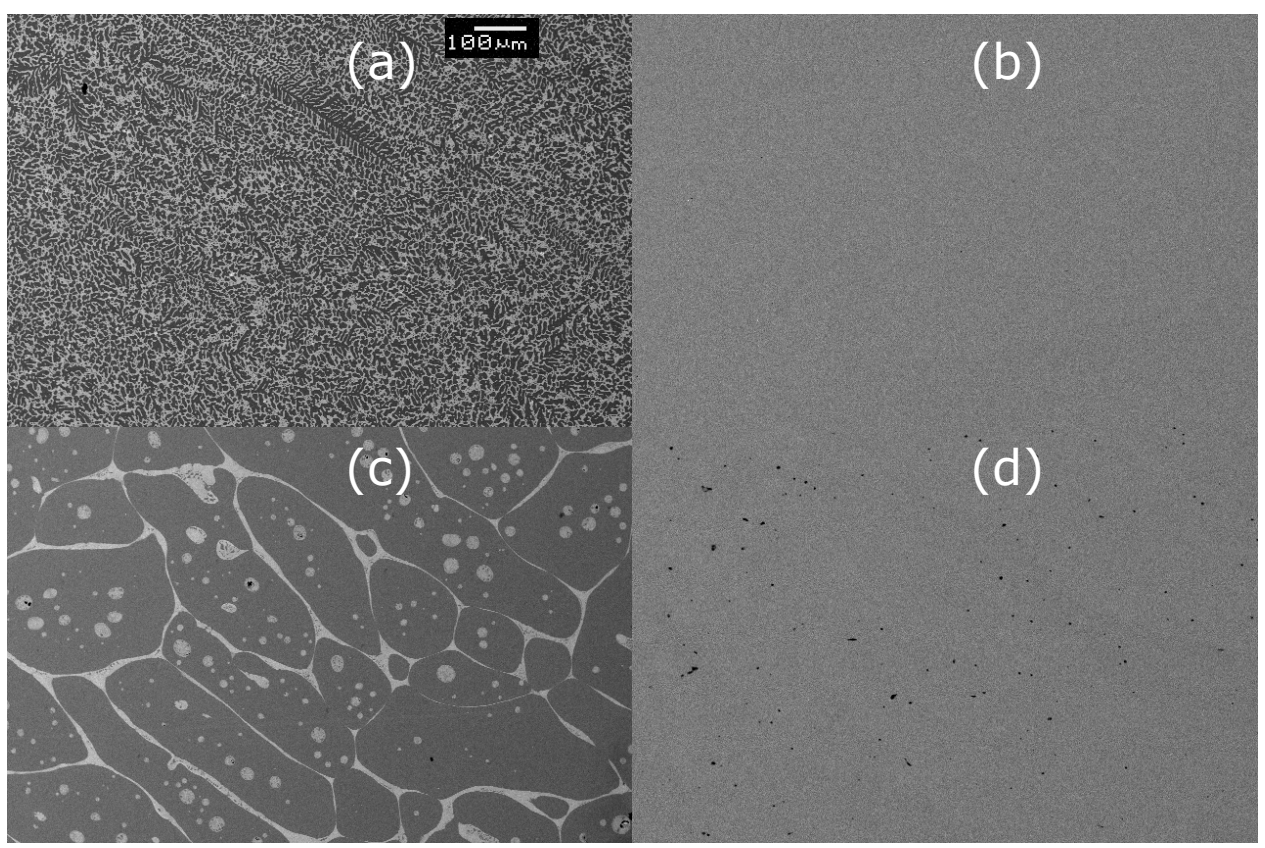

Figure 6: Representative SEM micrographs showing which ternary additions formed single phase or multi-phase microstructures. (a) Hf, (b) Pd, (c) Zr, and (d) Rh. All micrographs were obtained at 100x magnification using back scattered electrons.

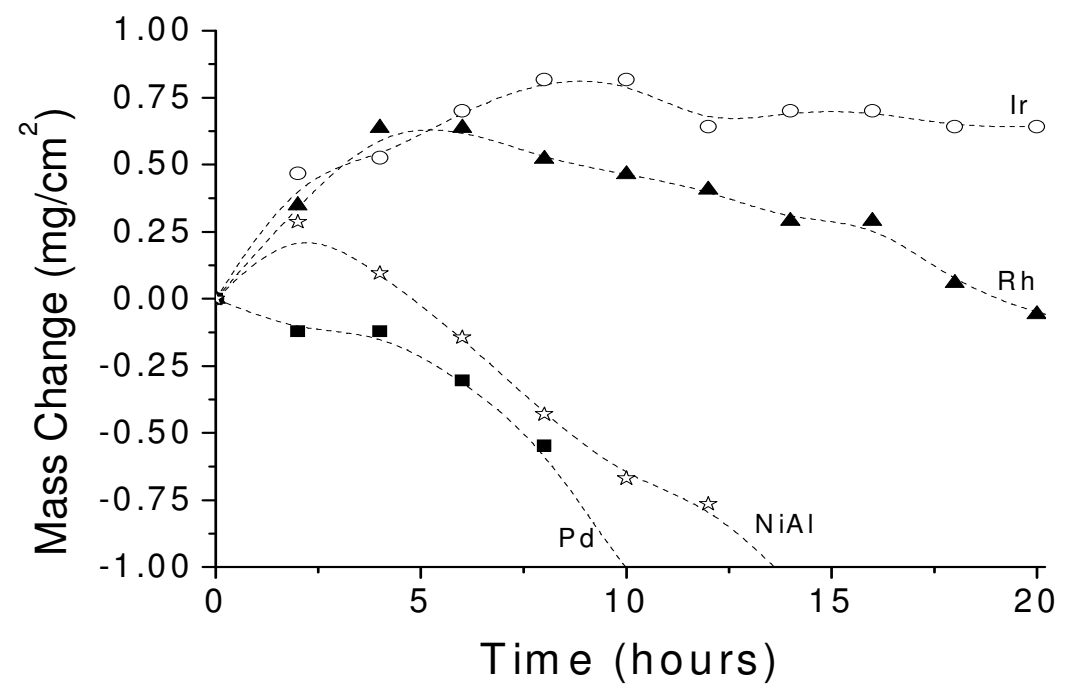

Figure 7: Cyclic oxidation of $\beta$-NiAl with and without PGM substitutions.

Figure 8a-d shows the oxidation microstructures of the baseline NiAl along with each ternary substitution set at 6 at $\%$. These samples were oxidized for 24 hours at $1300^{\circ} \mathrm{C}$ and then examined to better assess the evolution of the oxide scale formation. Figure 7 clearly displays that neither the benchmark NiAl alloy nor the NiAl doped with Pd additions show an adherent oxide layer. Since both alloys had relatively significant mass loss, the surface would be devoid of a continuous oxide scale. The Rh doped NiAl showed an overall mass gain as well as signs of 
some spallation. It can be seen that the oxide layer is fairly continuous with a thickness of approximately $10 \mu \mathrm{m}$. The Ir addition showed an almost continuous oxide scale with a thickness of 8 microns. The only discontinuities were observed at the corners of the specimen where stresses in the oxide would be highest.

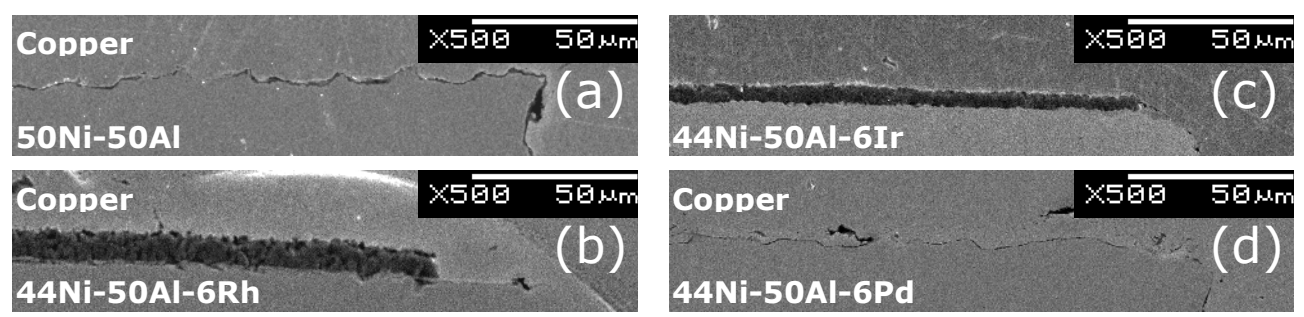

Figure 8a-d: Microstructures of oxidized alloys after oxidation at $1300^{\circ} \mathrm{C}$ for 24 hours. (a) $\beta$-NiAl; (b) $\beta$-NiAl with 6 atom percent $\mathrm{Rh}$ substituting $\mathrm{Ni}$, i.e. $\mathrm{Ni}_{44} \mathrm{Al}_{50} \mathrm{Rh}_{6}$ (c) $\beta$-NiAl with 6 atom percent Ir substituting Ni, i.e. $\mathrm{Ni}_{44} \mathrm{Al}_{50} \mathrm{Ir}_{6}$; (d) $\beta$-NiAl with 6 atom percent Pd substituting $\mathrm{Ni}$, i.e. $\mathrm{Ni}_{44} \mathrm{Al}_{50} \mathrm{Pd}_{6}$

\section{CONCLUSIONS}

We have shown that a hierarchical approach to alloy design starting with a less accurate but fast tool, followed by more accurate computational tools can be an efficient and effective means of down-selecting high temperature alloys. The modified Miedema model was used to identify regions of high (negative) formation enthalpies. Using the criteria of refractory base metal as the backbone of the alloy and identifying the NiAl as the most promising reservoir compound for alumina scale former, we demonstrated that Mo-Ni-Al shows good promise as a high temperature alloy. The most promising of the Ni-Al-(PGM) alloys based on ab inito calculations were synthesized and their oxidation behavior was studied. It was found that a few alloys in this system had enough potential to warrant further investigation of these materials for ultra-high temperature applications.

\section{REFERENCES}

[1] J. R. Nicholls, MRS Bulletin 28 (2003) 659-670.

[2] S. W. Wang, Oxidation of Metals 15 (1981) 1573.

[3] P. K. Ray, M. Akinc, M. J. Kramer, Journal of Alloys and Compounds 489 (2010) 357-361.

[4] G. Kresse, J. Furthmüller, Physical Review B 54 (1996) 11169.

[5] G. Kresse, J. Furthmüller, Computational Materials Science 6 (1996) 15-50.

[6] G. Kresse, J. Hafner, Physical Review B 47 (1993) 558.

[7] H. J. Monkhorst, J. D. Pack, Physical Review B 13 (1976) 5188.

[8] X. Lu, Y. Cui, Z. Jin, Metallurgical and Materials Transactions A 30 (1999) 1785-1795.

[9] B. H. Toby, Journal of Applied Crystallography 34 (2001) 210-213.

[10] G. R. Wallwork, Reports on Progress in Physics 39 (1976) 401-485.

[11] X. Zhao, I. P. Shapiro, P. Xiao, Surface and Coatings Technology 202 (2008) 2905-2916.

[12] M. K. Meyer, M. Akinc, Journal of the American Ceramic Society 79 (1996) 938-944. 